Homology, Homotopy and Applications, vol.18(2), 2016, pp.85-106

\title{
DUALITY FOR DIAGRAM CHASING A LA MAC LANE IN NON-ABELIAN CATEGORIES
}

\author{
ZURAB JANELIDZE \\ (communicated by Maria Cristina Pedicchio)
}

\begin{abstract}
In this paper we provide an axiomatic analysis of the classical diagram chasing method of Mac Lane, that relies on duality and chasing elements of pointed sets, which allows one to generalize this method from abelian categories to non-abelian ones. Among the examples where the generalized method can be used are all modular semiexact categories in the sense of Grandis (which include all Puppe-Mitchell exact categories) and sequentiable categories in the sense of Bourn (which include all semi-abelian categories, and in particular, the categories of group-like structures). The method turns out to be closely related to the essential features of these categories. At the same time, in some sense, it simplifies the usual proofs of some of the standard diagram lemmas in them.
\end{abstract}

\section{Introduction}

The so-called "diagram chasing" is a technique introduced by Mac Lane [24] for proving homological diagram lemmas in concrete categories of structures, and in general abelian categories. Since then this technique has been adapted to many different general contexts. In an abelian category, the technique is very efficient thanks to duality: by dualising a half of the proof of a diagram lemma one obtains the complete proof. This means that a category-theoretic proof of a homological diagram lemma in a concrete abelian category, such as the category of abelian groups, is in some sense more elegant than the set-theoretic proof involving elements. One of the main aims of this paper is to show that a similar statement can be made for some of the diagram lemmas in many of the principal non-abelian categories, which includes categories of group-like structures, and many more.

Half a century of developments, following the proposal for an axiomatic approach in the study of the category of groups made in [23], leads to the notion of a semi-abelian

Partially supported by South African National Research Foundation. The author is grateful for the kind hospitality of the University of Louvain-la-Neuve.

Received February 7, 2015, revised January 2, 2016; published on August 10, 2016.

2010 Mathematics Subject Classification: 18G50, 18D99, 18E10, 18A20, 18A32, 18C99, 20J15, $55 \mathrm{U} 30$.

Key words and phrases: $3 \times 3$ lemma, abelian category, diagram lemma, exact sequence, five lemma, homological algebra, homological category, modular semiexact category, pointed set, pointed subobject functor, protomodular category, regular category, sequentiable category, subobject chasing.

Article available at http://dx.doi.org/10.4310/HHA.2016.v18.n2.a5

Copyright (C) 2016, Zurab Janelidze. Permission to copy for private use granted. 
category [16]; semi-abelian categories are to categories of group-like and slightly more general structures (e.g., loops, groups, rings, algebras, modules, etc.), as abelian categories are to categories of modules. A semi-abelian category is a finitely complete Barr exact [1], Bourn protomodular [5] pointed category having binary sums. Abelian categories are precisely those semi-abelian categories whose duals are also semi-abelian. Thus, semi-abelian categories cannot exhibit as much duality as abelian categories can. Nevertheless, already in [29] it is shown that some of the diagram lemmas can be proved by duality in non-abelian categories such as the category of groups. This is perhaps not too surprising, since a short exact sequence in these categories, including all semi-abelian ones, can be still defined in a self-dual way. The present paper can be seen as a revival of this line of research, which builds on some of the more recent developments in categorical algebra, and at the same time, exposes some concealed links between these developments and observations concerning diagram lemmas in abelian categories due to Mac Lane.

In [19], it was shown that certain principal properties of semi-abelian categories can be given a self-dual form by representing a semi-abelian category $\mathbb{C}$ through a "projection" onto $\mathbb{C}$ - a functor from some category $\mathbb{B}$ to $\mathbb{C}$, and in particular, the right functor for this purpose is the Grothendieck bifibration $\mathbb{B} \rightarrow \mathbb{C}$ of subobjects of the semi-abelian category $\mathbb{C}$. By "duality" with respect to such structure we mean the usual duality for a functor, as defined in, e.g., [25], which takes dual of both categories, and replaces the functor with its dual functor $\mathbb{B}^{\text {op }} \rightarrow \mathbb{C}^{\text {op }}$. It seems that much of the non-dual theory of semi-abelian categories can be recovered from a more general self-dual theory developed in the context of a category equipped with a functor to it. This in some way resembles how projective plane geometry allows to develop a self-dual treatment of certain topics in Euclidean plane geometry (see, e.g., [28]). Another analogy with the ideas emerging from projective geometry is the role played by subobject lattices, which are usually the fibres of the functor required for the above purposes - see the Prefaces of the two recent monographs $[\mathbf{1 1}, \mathbf{1 2}]$ where Grandis explains why he calls his approach to homological algebra, based on subobject lattices (more precisely, lattices of normal subobjects), a "projective approach". It should be remarked, however, that the role of subobject lattices for similar purposes as we have in mind is in some sense hinted already in the very first paper [23] where duality of the context of a category is explored for the first time, and which later gave rise to the modern self-dual notion of an abelian category (for a brief history of the development of this notion, see the note to the chapter on abelian categories in [25]). Another place where the importance of viewing objects in a category via their subobjects is emphasized in the work of Mac Lane is in his subobject chasing technique [24]. In some sense, the present paper together with $[\mathbf{1 9}, \mathbf{2 0}, \mathbf{2 1}]$ show how these ideas can be brought together and merged with more recent developments in categorical algebra, giving rise to what should perhaps be called, adopting and extending the terminology of Grandis, a "projective approach to non-abelian algebra".

Let us briefly sketch Mac Lane's technique of chasing subobjects in an abelian category $\mathbb{C}$ (see $[\mathbf{2 4}, \mathbf{2 5}]$ ). It can be formalised as a list of preservation-reflection properties of a (covariant) functor $F: \mathbb{C} \rightarrow$ Set $_{*}$ defined as follows:

- for each object $X$ in $\mathbb{C}$, the elements of the pointed set $F(X)$ are subobjects of $X$, with the base point being the subobject given by a null morphism $0 \rightarrow X$; 
- for each morphism $f: X \rightarrow Y$ in $\mathbb{C}$, a subobject of $X$ given by a monomorphism $m: M \rightarrow X$ is mapped by $F(f)$ to the subobject of $Y$ given by the monomorphism $m^{\prime}$ in the decomposition $f m=m^{\prime} e$ of $f m$ into an epimorphism followed by a monomorphism ( $m^{\prime}$ is the "image" of $m$ under $\left.f\right)$.

We call $F$ the covariant pointed subobject functor, as in [17]. The required preservation-reflection properties of this functor are:

- a morphism $f: X \rightarrow Y$ in $\mathbb{C}$ is a null morphism if and only if $F(f)$ is a null morphism of pointed sets, i.e., $\operatorname{Im} F(f)=\{0\}$ (where 0 denotes the base point of the pointed set $F(Y)$ );

- it is an epimorphism if and only if $F(f)$ is surjective;

- it is a monomorphism if and only if $F(f)$ is injective, and if and only if $\operatorname{Ker} F(f)=$ $\{0\}$ (i.e., if $F(f)(x)=0$ then $x=0$ );

- a sequence

$$
X \stackrel{f}{\longrightarrow} Y \stackrel{g}{\longrightarrow} Z
$$

of morphisms in $\mathbb{C}$ is exact at $Y$ if and only if the corresponding sequence

$$
F(X) \stackrel{f}{\longrightarrow} F(Y) \stackrel{g}{\longrightarrow} F(Z)
$$

of morphisms of pointed sets is exact at $F(Y)$, i.e., $\operatorname{Im} F(f)=\operatorname{Ker} F(g)$.

These properties are established in Theorem 2.2 in $[\mathbf{2 4}]$. The covariant pointed subobject functor reduces diagram chasing in an abelian category, to chasing diagrams of pointed sets. Since the dual of an abelian category $\mathbb{C}$ is still an abelian category, we get a similar list of properties for the contravariant functor $\mathbb{C}^{\text {op }} \rightarrow$ Set $_{*}$ which assigns to an object $X$ the pointed set of quotients of $X$ in $\mathbb{C}$, with the base point given by the null quotient $X \rightarrow 0$. Now, exactness of a sequence in an abelian category $\mathbb{C}$ is equivalent to exactness of the same sequence in the dual category $\mathbb{C}$, and so sometimes it becomes possible to use the covariant pointed subobject functor for establishing one half of a diagram lemma, and the contravariant quotient functor, for the other half. When these two halves are dual statements, the proof of the first half is sufficient to prove the entire lemma. Moreover, often one of the two dual halves of a diagram lemma requires subtraction and addition of elements/subobjects, while the other one does not. In these cases, proving the "easier" dual half suffices to prove the entire lemma. This advantage of proving diagram lemmas in the context of an abelian category is in fact inherent also in non-abelian contexts, as we show in the present paper.

Note that in an abelian category $\mathbb{C}$, via the standard kernel-cokernel correspondence, quotients of $X$ can be identified with subobjects of $X$ (in an abelian category, any subobject is normal), and then the dual of the covariant pointed subobject functor becomes the contravariant pointed subobject functor $F^{\prime}: \mathbb{C}^{\mathrm{op}} \rightarrow \mathbf{S e t}_{*}$. Mac Lane's diagram chasing technique becomes then based on the properties listed above, of the two functors:

$$
F: \mathbb{C} \rightarrow \mathbf{S e t}_{*}, \quad F^{\prime}: \mathbb{C}^{\mathrm{op}} \rightarrow \mathbf{S e t}_{*} .
$$

It turns out that abelianness of the category $\mathbb{C}$ here is less significant, and the power of the diagram chasing technique lies essentially in the fact that the two functors above 
agree with each other in a suitable sense. As we will see, similar pairs of functors can be constructed also for the categories studied in non-abelian algebra, and hence we get a new simplified approach to proving some of the standard diagram lemmas in these categories. In particular, this is the case for all sequentiable categories in the sense of Bourn [6] (which include the above-mentioned semi-abelian categories), as well as for all modular semiexact categories in the sense of Grandis $[\mathbf{1 0}, \mathbf{1 2}]$.

A general analysis of Mac Lane's subobject chasing technique in terms of the covariant pointed subobject functor, and the corresponding generalization to nonabelian categories, has already been carried out in $[\mathbf{1 7}, \mathbf{1 8}]$. However, the theory developed there is not a self-dual one. The new approach explored in the present work is to consider both the covariant and the contravariant pointed subobject functors simultaneously - this enforces the theory with duality, similar to the one present in the classical context of an abelian category.

It must be made clear that the intention of this work is not to provide a general axiomatic context for treating all diagram lemmas of homological algebra. Instead, our aim is to identify those lemmas whose proofs in the classical abelian case can be carried out by arguments that rely solely on chasing elements of pointed sets and duality. For instance, we show by a counterexample that the "middle $3 \times 3$ lemma" cannot be proved using our technique. However, we do not exclude the possibility of a future specialization of the context we propose in the present paper, still general enough not to exclude principal examples, that would encompass all desirable diagram lemmas.

This work leads also to some other open questions and conjectures. For instance, there is an impression that any diagram lemma that can be proved for abelian groups can be also proved for non-abelian groups. It seems as well that any diagram lemma that can be proved for abelian groups, where the hypothesis is just exactness of sequences (and not that a certain composite of morphisms is a null morphism, as in the middle $3 \times 3$ lemma), and the conclusion is of the same type (and does not include existence of a new morphism, as in the snake lemma), can be also proved in the general context considered in this paper.

The paper is organized in two sections, apart from this introduction. In the first section, we develop the general theory and in the second section we discuss examples and applications. The first section requires minimal knowledge of category theory. We assume only that the reader is familiar with the notions of category and functor, as introduced in [8]. In the second section, we assume that the reader is familiar with basic material on categories from [25]; while some other basic concepts from category theory that are required to understand a large part of the section have been recalled in sufficient detail (e.g., the notion of a regular category [1]), and some parts are most elementary, an in-depth understanding of a few remaining parts substantially rely on the cited literature (and in a few places, knowledge of the basic theory of Grothendieck fibrations $[\mathbf{1 4}, \mathbf{1 5}])$.

\section{General theory}

Preliminaries 1.1 (Zero object; exact sequence of pointed sets). Recall from [25] that a zero object in a category is an object which admits a unique morphism to any other object, and a unique morphism from any other object, i.e., it is an object which 
is both initial and terminal. In particular, in the category Set $_{*}$ of pointed sets, such object exists and is given by a singleton pointed set. We denote it by $\mathbf{0}$.

For a pointed set $X$, we write $*_{X}$ to denote its base point. A morphism of pointed sets is a constant map if and only if it factors through the zero object $\mathbf{0}$. A sequence

$$
A \stackrel{u}{\longrightarrow} B \stackrel{v}{\longrightarrow} C
$$

of morphisms in $\mathbf{S e t}_{*}$ is exact when $\operatorname{Ker} v=\operatorname{Im} u$, where $\operatorname{Im} u=\{u(a) \mid a \in A\}$ and $\operatorname{Ker} v=\left\{b \in B \mid v(b)=*_{X}\right\}$.

Conditions 1.2. For a pair of functors

$$
(-)_{*}: \mathbb{C} \rightarrow \text { Set }_{*}, \quad(-)^{*}: \mathbb{C}^{\text {op }} \rightarrow \text { Set }_{*},
$$

we consider the following conditions:

(D1) For a sequence

$$
X \stackrel{f}{\longrightarrow} Y \stackrel{g}{\longrightarrow} Z
$$

of morphisms in $\mathbb{C}$, the sequence

$$
X_{*} \stackrel{f_{*}}{\longrightarrow} Y_{*} \stackrel{g_{*}}{\longrightarrow} Z_{*}
$$

is exact in Set $_{*}$ if and only if the sequence

$$
Z^{*} \stackrel{g^{*}}{\longrightarrow} Y^{*} \stackrel{f^{*}}{\longrightarrow} X^{*}
$$

is exact in Set $_{*}$.

(D2) For a morphism $f: X \rightarrow Y$ in $\mathbb{C}$, the following conditions are equivalent: (a) $f_{*}$ is injective; (b) the sequence

$$
\mathbf{0} \longrightarrow X_{*} \stackrel{f_{*}}{\longrightarrow} Y_{*}
$$

is exact in $\mathbf{S e t}_{*}$; (c) $f^{*}$ is surjective, or equivalently, the sequence

$$
Y^{*} \stackrel{f^{*}}{\longrightarrow} X^{*} \longrightarrow \mathbf{0}
$$

is exact in Set $_{*}$. Similarly, the following conditions are equivalent: (d) $f^{*}$ is injective; (e) the sequence

$$
\mathbf{0} \longrightarrow Y^{*} \stackrel{f^{*}}{\longrightarrow} X^{*}
$$

is exact in $\mathbf{S e t}_{*}$; (f) $f_{*}$ is surjective, or equivalently, the sequence

$$
X_{*} \stackrel{f_{*}}{\longrightarrow} Y_{*} \longrightarrow 0
$$

is exact in Set $_{*}$.

(D3) For a morphism $f: X \rightarrow Y$ in $\mathbb{C}$, the map $f_{*}$ is a bijection if and only if $f^{*}$ is a bijection and if and only if $f$ is an isomorphism.

Definition 1.3. A diagram chasing context consists of a category $\mathbb{C}$ equipped with a pair of functors as above satisfying (D1) and (D2). When (D3) is also satisfied then the diagram chasing context is called a strict diagram chasing context. 
Objects and morphisms of a diagram chasing context are those of the ground category $\mathbb{C}$. In a diagram chasing context, a sequence (1) of morphisms is said to be an exact sequence, when the corresponding sequences displayed in (D1) are exact in Set $_{*}$.

Lemma 1.4. For a morphism $f: X \rightarrow Y$ in a diagram chasing context the following conditions are equivalent:

(a) The sequence

$$
X \stackrel{f}{\longrightarrow} Y \stackrel{1_{Y}}{\longrightarrow} Y
$$

is exact.

(b) Both $f_{*}$ and $f^{*}$ are constant maps.

(c) $f_{*}$ is a constant map or $f^{*}$ is constant map.

(d) The sequence

$$
X \stackrel{1_{X}}{\longrightarrow} X \stackrel{f}{\longrightarrow} Y
$$

is exact.

Proof. This follows from (D1) and the fact that when $f$ is a morphism of pointed sets, both (a) and (d), with exactness there meaning the usual exactness for morphisms of pointed sets recalled above, are equivalent to $f$ being a constant map.

Definition 1.5. A morphism $f$ in a diagram chasing context is said to be a null morphism if it satisfies the equivalent conditions $1.4(\mathrm{a})-(\mathrm{d})$.

Remark 1.6. If for every object $X$ there exists a null morphism with codomain $X$, then, thanks to (D1), the condition (b) in (D2) can be dropped. Indeed, in this case the sequence in (b) can be replaced with the sequence

$$
W_{*} \stackrel{e_{*}}{\longrightarrow} X_{*} \stackrel{f_{*}}{\longrightarrow} Y_{*},
$$

where $e$ is a null morphism with codomain $X$. By (D1), exactness of this sequence is equivalent to exactness of the sequence

$$
Y^{*} \stackrel{f^{*}}{\longrightarrow} X^{*} \stackrel{e^{*}}{\longrightarrow} W^{*}
$$

and since $e^{*}$ here is a constant map, exactness of the above sequence becomes equivalent to surjectivity of $f^{*}$, which gives us (c).

Similarly, if for every object $X$ there exists a null morphism with domain $X$, then the condition (e) in (D2) can be dropped.

Definition 1.7. An object $X$ in a diagram chasing context is a null object when $1_{X}$ is a null morphism. A morphism $f: X \rightarrow Y$ in a diagram chasing context is said to be an equivalence when $f_{*}$ is a bijection, or equivalently, when $f^{*}$ is a bijection.

Lemma 1.8. For an object $X$ in a diagram chasing context the following conditions are equivalent:

(a) $1_{X}$ is a null morphism. 
(b) Both $X_{*}$ and $X^{*}$ are singletons, where by a "singleton" we mean a pointed set which is isomorphic to $\mathbf{0}$, i.e., a pointed set whose base point is its unique element.

(c) $X_{*}$ is a singleton or $X^{*}$ is a singleton.

Proof. This is a trivial consequence of the fact that a pointed set is a singleton if and only if its identity morphism is a constant map.

Remark 1.9. Note that in a diagram chasing context every isomorphism is an equivalence. Axiom (D3) states that the converse is also true.

In a diagram chasing context we can carry out many of the diagram chasing arguments in an essentially identical way as we would in the case of the context of an abelian category, relying on duality. We illustrate this by proving the so-called "five lemma" below.

Lemma 1.10 (Five lemma in a diagram chasing context). In a diagram chasing context, for any commutative diagram

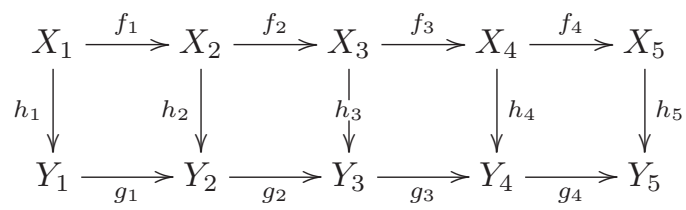

where the horizontal rows are exact sequences, if $h_{1}, h_{2}, h_{4}, h_{5}$ are equivalences, then $h_{3}$ is an equivalence. In a strict diagram chasing context, we can also conclude that $h_{3}$ is an isomorphism.

Proof. To show that $h_{3}$ is an equivalence, it suffices to show that the sequence

$$
\mathbf{0} \longrightarrow\left(X_{3}\right)_{*} \stackrel{\left(h_{3}\right)_{*}}{\longrightarrow}\left(Y_{3}\right)_{*} \longrightarrow \mathbf{0}
$$

is exact. As it is classically done in the context of an abelian category (see, e.g., [25]), we will first show exactness of this sequence at $\left(X_{3}\right)_{*}$, and deduce exactness at $\left(Y_{3}\right)_{*}$ by duality. After applying $(-)_{*}$ to the entire diagram in the lemma, we can carry out the following standard diagram chase:

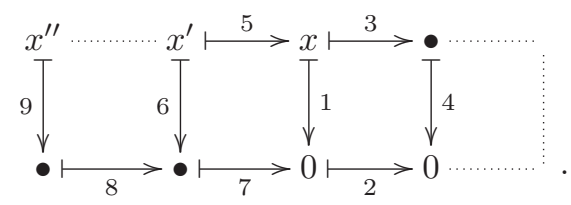

Commutativity of the diagram alone does not force $\left(f_{1}\right)_{*} x^{\prime \prime}=x^{\prime}$. However, it does force $\left(h_{2}\right)_{*}\left(f_{1}\right)_{*} x^{\prime \prime}=\left(h_{2}\right)_{*} x^{\prime}$, which implies that $\left(f_{1}\right)_{*} x^{\prime \prime}=x^{\prime}$. After this, $x=0$, which shows exactness of the above sequence at $\left(X_{3}\right)_{*}$. A symmetric argument, using the functor $(-)^{*}$ in the place of $(-)_{*}$, will prove exactness of the sequence

$$
\mathbf{0} \longrightarrow\left(Y_{3}\right)^{*} \stackrel{\left(h_{3}\right)^{*}}{\longrightarrow}\left(X_{3}\right)^{*} \longrightarrow \mathbf{0}
$$

at $\left(Y_{3}\right)^{*}$, which is equivalent to exactness of the previous sequence at $\left(Y_{3}\right)_{*}$. 
Remark 1.11 (Other diagram lemmas in a diagram chasing context). We must remark that in a diagram chasing context, as defined above, we expect to be able to establish only those diagram lemmas which in an abelian category could be proved, using only elementary diagram chasing arguments, avoiding subtraction or addition of elements, thanks to duality. Among such diagram lemmas are, apart from the five lemma and its various partial formulations, also the upper and the lower $3 \times 3$ lemmas, and exactness of the long sequence in the snake lemma, once a suitable connecting morphism has been given (see [18]). The "cross lemma" in the sense of [18] can be also proved in a diagram chasing context. It is not difficult to invent new diagram lemmas that can be proved in a diagram chasing context. For instance, such is the "dragon lemma" which states that if all sequences in the diagram below are exact, then so are the sequences $0 \rightarrow X \rightarrow Y$ and $B \rightarrow A \rightarrow 0$.
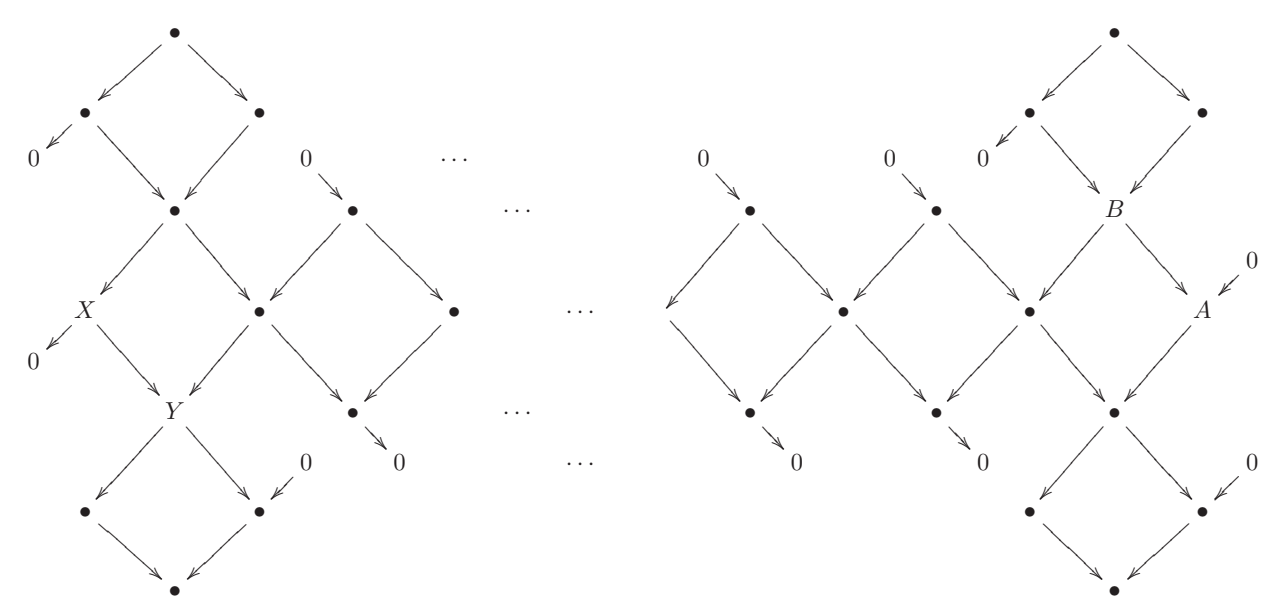

To prove exactness of the sequence $0 \rightarrow X \rightarrow Y$, apply the functor $(-)_{*}$ to the diagram and chase elements from the left "head" of the dragon to its right "head" and then back, along to top part of the dragon. Exactness of the sequence $B \rightarrow A \rightarrow 0$ follows by duality.

It turns out that the middle $3 \times 3$ lemma cannot be proved in a diagram chasing context. We show this below by constructing a counterexample.

Definition 1.12. In a diagram chasing context, define a short exact sequence to be a sequence

$$
X \stackrel{f}{\longrightarrow} Y \stackrel{g}{\longrightarrow} Z
$$

of morphisms such that the sequence

$$
\mathbf{0} \longrightarrow X_{*} \stackrel{f_{*}}{\longrightarrow} Y_{*} \stackrel{g_{*}}{\longrightarrow} Z_{*} \longrightarrow \mathbf{0}
$$

is exact in $\mathbf{S e t}_{*}$, or equivalently, the sequence

$$
\mathbf{0} \longrightarrow Z^{*} \stackrel{g^{*}}{\longrightarrow} Y^{*} \stackrel{f^{*}}{\longrightarrow} X^{*} \longrightarrow \mathbf{0}
$$


is exact in $\mathbf{S e t}_{*}$. The middle $3 \times 3$ lemma states that in a commutative diagram

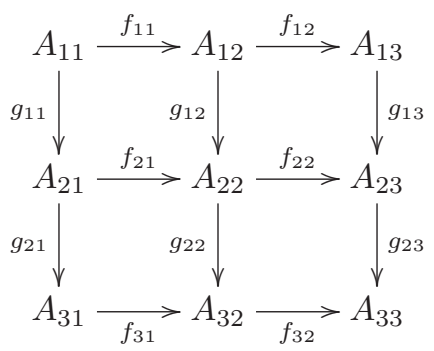

if the composite of morphisms in the middle row is a null morphism, and the remaining rows are short exact sequences, as well as all three columns, then the middle row is also a short exact sequence.

Counterexample 1.13. [A diagram chasing context where the middle $3 \times 3$ lemma fails] We now show that the middle $3 \times 3$ lemma is not necessarily true, even in a strict diagram chasing context. In the middle $3 \times 3$ lemma as formulated above, one requires that the sequence

$$
\mathbf{0} \longrightarrow\left(A_{21}\right)_{*} \stackrel{\left(f_{21}\right)_{*}}{\longrightarrow}\left(A_{22}\right)_{*} \stackrel{\left(f_{22}\right)_{*}}{\longrightarrow}\left(A_{23}\right)_{*} \longrightarrow \mathbf{0}
$$

is exact. It is easy to prove exactness at $\left(A_{21}\right)_{*}$, and by duality, deduce exactness at $\left(A_{23}\right)_{*}$. However, exactness at $\left(A_{22}\right)_{*}$ cannot be established. To show this, consider the free category $\mathbb{C}$ over the graph

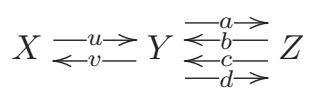

satisfying the identities

$$
v u=1_{X}, \quad c a=u v=b d, \quad b a=1_{Y}=c d, \quad a u=d u, \quad v b=v c .
$$

Notice that this system of identities is invariant under interchanging $u$ with $v, a$ with $c$, and $b$ with $d$ and then changing the order of composition. This gives rise to an isomorphism of categories $I: \mathbb{C}^{\text {op }} \rightarrow \mathbb{C}$, whose inverse is its dual, which is identity on objects, and under which $a \mapsto c$ and $b \mapsto d$. We will use this isomorphism below.

The next step is to remark that the category $\mathbb{C}$ has 19 morphisms, with the composition given in Table 1. The identities corresponding to those which we used to define the category $\mathbb{C}$ hold for the diagram

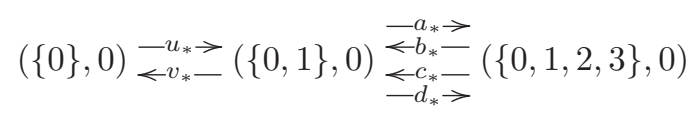

of pointed sets, where $u_{*}$ and $v_{*}$ are uniquely determined, while the other maps are defined as follows:

$$
\begin{gathered}
a_{*}(x)=x, \\
b_{*}(x)= \begin{cases}1, & x=1, \\
0, & x \in\{0,2,3\},\end{cases} \\
c_{*}(x)= \begin{cases}1, & x \in\{2,3\}, \\
0, & x \in\{0,1\},\end{cases}
\end{gathered}
$$




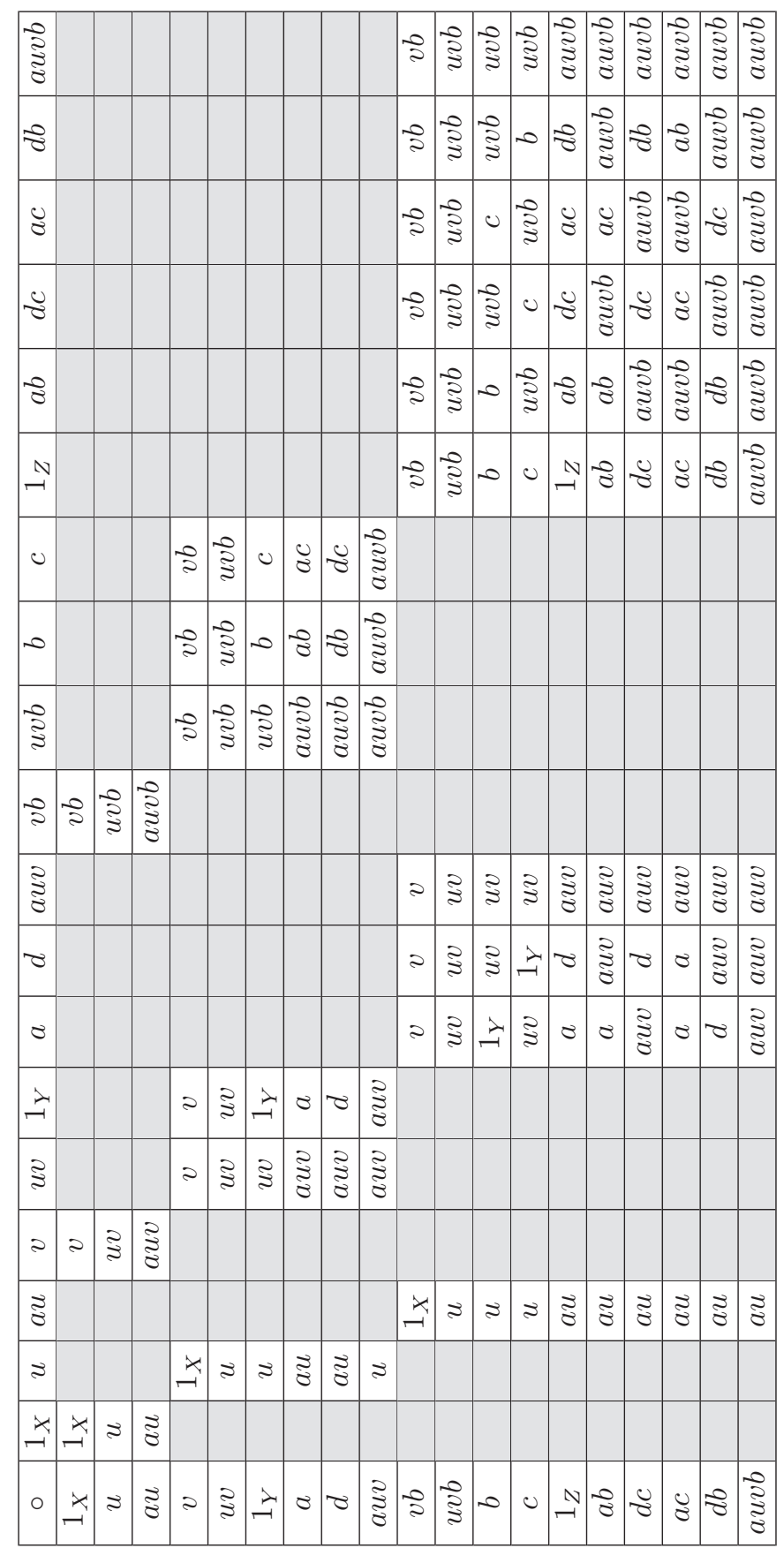

Table 1: Composition table for the category in Counterexample 1.13 


$$
d_{*}(x)=2 x .
$$

Thus, the above diagram determines a functor $(-)_{*}: \mathbb{C} \rightarrow \mathbf{S e t}_{*}$. Similarly, the functor $(-)^{*}=I(-)_{*}=(-)_{*} \circ I: \mathbb{C}^{\mathrm{op}} \rightarrow$ Set $_{*}$, where $I$ is the isomorphism defined above, is determined by the diagram

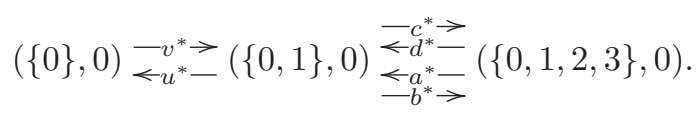

With the help of Table 2, and the display given below, it is a simple routine to verify that this pair of functors constitutes a strict diagram chasing context.

In the following display, consider the diagram
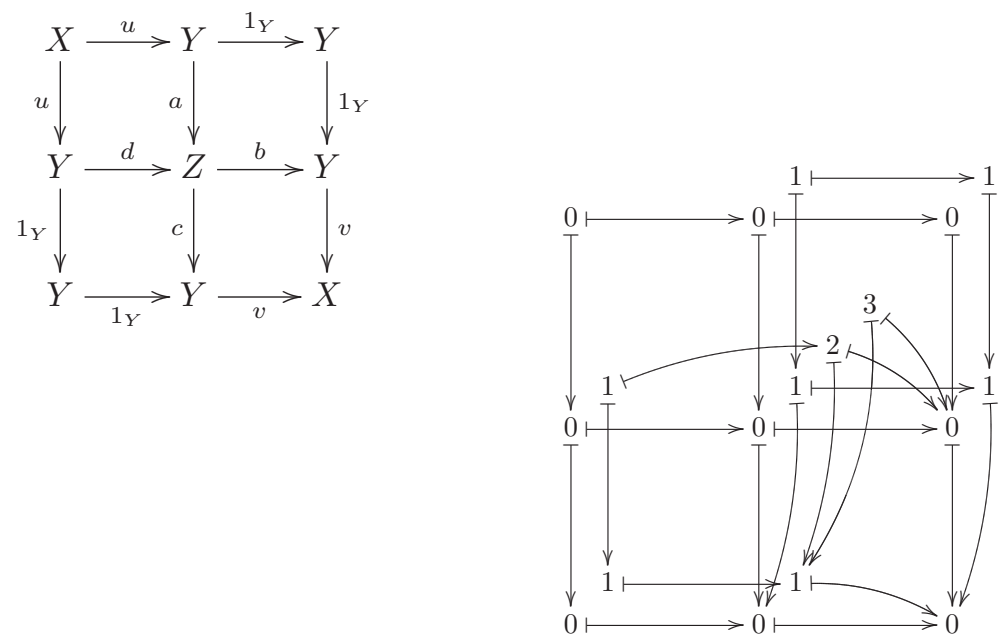

at the top left corner, seen as a diagram in $\mathbb{C}$. This diagram meets all assumptions stated in the middle $3 \times 3$ lemma, but the middle sequence is not an exact sequence. Hence, the middle $3 \times 3$ lemma fails in the strict diagram chasing context that we have constructed. The figure in the bottom right corner of the above display sketches the image of the previous diagram under the functor $(-)_{*}$; to obtain the image of the same diagram under the functor $(-)^{*}$, simply rotate this figure by $180^{\circ}$ around its centre.

\section{Examples and applications}

Preliminaries 2.1 (Subobjects in regular categories). The class of regular protomodular categories is a wider class of categories than the class of semi-abelian categories. Hence, they include categories of various group-like structures, as well as the category of, say, topological groups which is not a semi-abelian category. As we will see below, functorial analysis of diagram chasing brings us very close to the essence of this class of categories.

Recall that a regular category is a finitely complete category $\mathbb{C}$ (i.e., a category having finite limits), in which any morphism $f$ can be factorized $f=m e$ as a pullbackstable regular epimorphism $e$ followed by a monomorphism $m$. Recall that a regular epimorphism is a coequalizer of a parallel pair of morphisms. A pullback-stable regular 


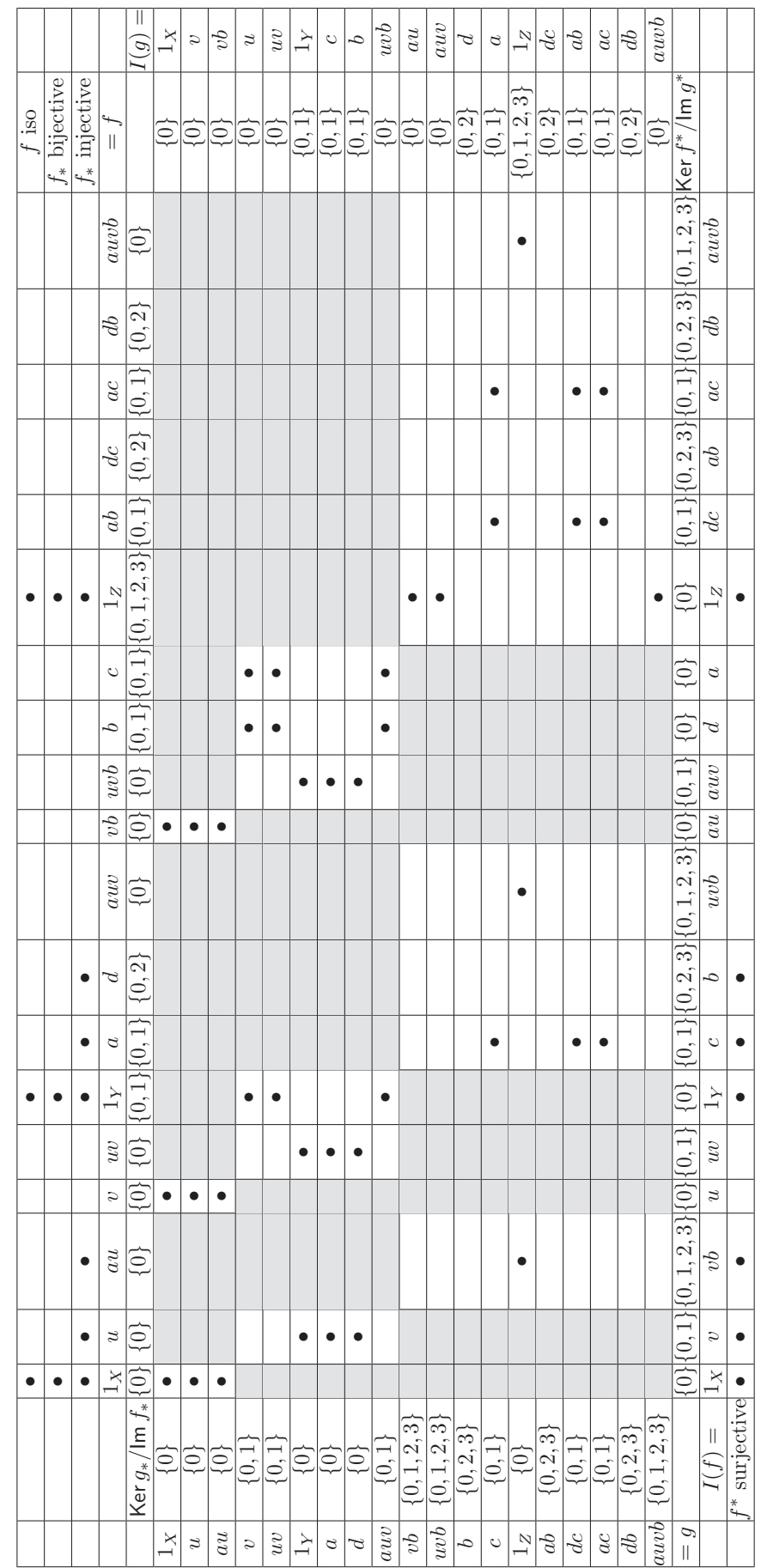

Table 2: The two functors in Counterexample 1.13 constitute a strict diagram chasing context 
epimorphism is a regular epimorphism whose pullback along any morphism is still a regular epimorphism. In the familiar regular categories of structures (e.g., categories of groups, rings, etc.), regular epimorphisms are just surjective structure-preserving maps. Just as in these categories, in any regular category, any regular epimorphism is a pullback-stable regular epimorphism. Also, in a regular category the class of regular epimorphisms is closed under composition (in general, the class of pullback-stable regular epimorphisms is closed under composition in any category with pullbacks).

Recall that a subobject of an object $X$ (in any category $\mathbb{C}$ ) is an equivalence class of monomorphisms $m: M \rightarrow X$, under the equivalence relation which identifies two monomorphisms $m$ and $m^{\prime}$ when they factor through each other, i.e., $m=m^{\prime} j$ and $m^{\prime}=m j^{\prime}$, for some morphisms $j$ and $j^{\prime}$; when this happens, the morphisms $j$ and $j^{\prime}$ in the factorizations are unique, and are both isomorphisms, being inverses of each other. When talking about a subobject we often write one of the members of the equivalence class. For a morphism $f: X \rightarrow Y$ in a regular category, the subobject of $Y$ defined by the monomorphism $m$ in a factorization $f=m e$ where $e$ is a regular epimorphism, is invariant under the factorization and is called the image of $f$. The direct image of a subobject $m: M \rightarrow X$ under a morphism $f$ is defined as the image of the composite $\mathrm{fm}$. The inverse image of a subobject $m: M \rightarrow X$ under a morphism $g: W \rightarrow X$ is defined as the subobject given by the pullback of the morphism $m$ along the morphism $g$. Both of these constructions are well defined, i.e., they are invariant under each choice made in the construction (the choice of a monomorphism to represent the subobject, as well as the choice of a pullback). All of these notions obtain the expected meaning in "familiar regular categories" referred to above.

Subobjects of an object can be ordered under factorization of the monomorphisms that represent them: we say that a subobject $m: M \rightarrow X$ of an object $X$ is smaller than another subobject $m^{\prime}: M^{\prime} \rightarrow X$, when $m$ factors through $m^{\prime}$, i.e., $m^{\prime} j=m$ for some morphism $j: M \rightarrow M^{\prime}$. This order relation is well-defined for each object $X$ and is in fact a partial order, i.e., it is reflexive, transitive and antisymmetric. As expected, every morphism $f: X \rightarrow Y$ gives rise to a Galois connection between the partially ordered sets (classes) of subobjects of $X$ and subobjects of $Y$, via the direct and inverse image along $f$ mappings. Note that every object $X$ in a regular category has a greatest subobject, given by the monomorphism $1_{X}: X \rightarrow X$ (or any isomorphism with codomain $X$ ). The least subobject may not always exist, and when it does we say that the regular category has least subobjects. This is the case, for instance, when the regular category has an initial object 0 . Then, the least subobject of $X$ is the image of the unique morphism $0 \rightarrow X$.

For a regular category $\mathbb{C}$ having least subobjects, we can define the so-called covariant and the contravariant pointed subobject functors,

$$
(-)_{*}: \mathbb{C} \rightarrow \text { Set }_{*}, \quad(-)^{*}: \mathbb{C}^{\mathrm{op}} \rightarrow \text { Set }_{*},
$$

as follows:

- For an object $X$ in $\mathbb{C}$, the elements of the pointed set $X_{*}$ are subobjects of $X$, and the base point is the least subobject of $X$. The pointed set $X^{*}$ has the same elements, but its base point is the greatest subobject of $X$.

- For a morphism $f: X \rightarrow Y$ in $\mathbb{C}$, the function $f_{*}: X_{*} \rightarrow Y_{*}$ maps a subobject of $X$ to its direct image under $f$, and the function $f^{*}: Y^{*} \rightarrow X^{*}$ maps a subobject of $Y$ to its inverse image under $f$. 
Theorem 2.2. For a regular category $\mathbb{C}$ with least subobjects, the covariant and the contravariant pointed subobject functors constitute a diagram chasing context if and only if $\mathbb{C}$ is a protomodular category in the sense of Bourn [5]. If $\mathbb{C}$ has an initial object, then the same pair of functors constitute a strict diagram chasing context if and only if $\mathbb{C}$ is a sequentiable category in the sense of Bourn [6].

Proof. As shown in [19], a regular category is protomodular if and only if whenever a subobject $x$ of an object $X$ is larger than an inverse image $f^{*}(y)$ of some subobject $y$ of $Y$, along a morphism $f: X \rightarrow Y$, the subobject $x$ itself is an inverse image of some subobject of $y$; notice that since inverse and direct image maps form a Galois connection, we can equivalently require $x=f^{*} f_{*}(x)$. Under the presence of least subobjects, it is enough to ask this for $y$ being the least subobject $0_{Y}$ of $Y$. Following [19], we call this property right cartesianness of $f$. As remarked in [19], the dual property of left cartesianness of $f$, which states that if $x$ is smaller than the image of a morphism $g: W \rightarrow X$ (i.e., the direct image of the largest subobject of $W$ under $g$ ), then $x=g_{*} g^{*}(x)$, holds in any regular category; this can be confirmed by the following construction of the inverse image of $x$, where monomorphisms representing relevant subobjects have been labelled by the subobjects:

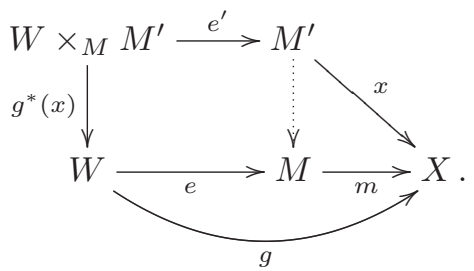

Ignore for now the label for the left-most arrow. $g=m e$ is the factorization of $g$ as a regular epimorphism $e$ followed by a monomorphism $m$. Thus, $m$ represents the image of $g$, and since $x$ is smaller than the image of $g$, we get the dotted arrow above. Consider a pullback rectangle on the left hand side. Since $e$ is a regular epimorphism, so must be $e^{\prime}$ (pullback-stability of regular epimorphisms). The fact that $m$ is a monomorphism implies that the outer trapezium is also a pullback. So, the left-most arrow does indeed represent the inverse image of $x$ under $g$, as the label indicates. Since $x$ is a monomorphism and $e^{\prime}$ is a regular epimorphism, we see that the direct image of $g^{*}(x)$ along $g$ coincides with $x$.

To prove the first part of the theorem, we begin by showing that the validity of the implication

$$
\operatorname{Ker} g_{*}=\operatorname{Im} f_{*} \quad \Rightarrow \quad \operatorname{Ker} f^{*}=\operatorname{Im} g^{*}
$$

(for sequences (1)) in (D1) is already equivalent to the above criterion for protomodularity (i.e., right cartesianness of every morphism), while the reverse implication as well as (D2) can be deduced from it. We begin by analyzing each of the equalities in the implication above:

- $\operatorname{Ker} g_{*}$ consists of all those subobjects $m: M \rightarrow Y$ of the object $Y$ from the display (1), such that $g_{*}(m)$ is the least subobject $0_{Z}$ of $Z$. It is thus the downclosure of $g^{*}\left(0_{Z}\right)$. On the other hand, the image of $f$, which can be obtained as the direct image $f_{*}\left(1_{X}\right)$ of $1_{X}$ under $f$, is the greatest element in $\operatorname{Im} f_{*}$ and so by 
left cartesianness of $f, \operatorname{Im} f_{*}$ is the down-closure of $f_{*}\left(1_{X}\right)$. Thus, the equality $\operatorname{Ker} g_{*}=\operatorname{Im} f_{*}$ is equivalent to $g^{*}\left(0_{Z}\right)=f_{*}\left(1_{X}\right)$.

- Ker $f^{*}$ consists of those subobject $m$ of $Y$, such that $f^{*}(m)$ is the largest subobject of $X$. Thus, it is the up-closure of $f_{*}\left(1_{X}\right)$. It then follows that the equality Ker $f^{*}=\operatorname{Im} g^{*}$ is equivalent to having $g^{*}\left(0_{Z}\right)=f_{*}\left(1_{X}\right)$ and $g$ being right cartesian.

From this we can conclude that (2) states that every morphism $g$ such that $g^{*}\left(0_{Z}\right)=$ $f_{*}\left(1_{X}\right)$ for some morphism $f$, is right cartesian. Since we can always find such $f$ (simply take $f$ to be a monomorphism which represents the subobject $g^{*}\left(0_{Z}\right)$ ), the implication is equivalent to every morphism being right cartesian, and hence to protomodularity.

The reverse implication to (2) holds trivially, after the above analysis of the equalities in it. The equivalences $(\mathrm{a}) \Leftrightarrow(\mathrm{c})$ and $(\mathrm{d}) \Leftrightarrow(\mathrm{f})$ in (D2) hold because $f_{*}$ and $f^{*}$ form a Galois connection between ordered sets of subobjects. The equivalence $(b) \Leftrightarrow(c)$ is due to right cartesianness and the equivalence $(\mathrm{e}) \Leftrightarrow(\mathrm{f})$ is due to left cartesianness.

Recall that a sequentiable category is a regular protomodular category with an initial object 0 , such that the unique morphism $0 \rightarrow 1$ from an initial object 0 to a terminal object 1 is a monomorphism. This gives rise to well-behaved notions of kernel and cokernel, which extend the usual notions of kernel and cokernel from a pointed category, see [6]. To see that (D3) holds in a sequentiable category, first note that already in any regular category, $f_{*}$ being surjective implies that $f_{*}$ is a regular epimorphism. As shown in [6], in a sequentiable category any regular epimorphism is a cokernel of its kernel. $f_{*}$ being injective gives that its kernel is trivial, and hence $f$ must be an isomorphism. Conversely, if (D3) holds in a regular protomodular category with an initial object 0 , then we can decompose the unique morphism $0 \rightarrow 1$,

$$
0 \stackrel{e}{\longrightarrow} H \stackrel{m}{\longrightarrow} 1 \text {, }
$$

into a regular epimorphism $e$ followed by a monomorphism $m$, and note that since the initial object 0 has only one subobject, the sequence

$$
0 \longrightarrow 0 \stackrel{e}{\longrightarrow} H
$$

is exact. This implies that $e_{*}$ is injective by (D2). At the same time, being a regular epimorphism, by left cartesianness, $e_{*}$ is surjective. So $e_{*}$ is a bijection, and hence $e$ is an isomorphism by (D3). Then, since $m$ is a monomorphism, so must be the composite me, which concludes the proof.

Examples 2.3 (Regular protomodular categories with least subobjects). An example of a regular protomodular category with an initial object 0 which is not a sequentiable category, is the dual of the category of sets. A sequentiable category in which the unique morphism $0 \rightarrow 1$ is an isomorphism (which is the same as a pointed regular protomodular category), is called a homological category in [4]. For an extensive list of examples of sequentiable and homological categories, see $[\mathbf{6}, 4]$. Among these categories are all usual pointed categories of group-like structures, and more generally, all semi-abelian categories (which include all abelian categories). In particular, the category of groups is one of such categories. The fact that any homomorphism of groups is cartesian (in the sense recalled in the proof of Theorem 2.2) is of course one of the basic facts about groups — see, e.g., Theorem II.22 in [2]. 
Corollary 2.4. A homological category in the sense of [4] is the same as a pointed regular category $\mathbb{C}$ for which the covariant and the contravariant pointed subobject functors constitute a (strict) diagram chasing context.

Remark 2.5 (Application). Theorem 2.2 describes a large class of examples of diagram chasing contexts. At the same time, it can be seen as an "application" of diagram chasing contexts, as it shows that, in some sense, a sequentiable category is precisely the type of regular category having least subobjects to which the subobject chasing technique from abelian categories, relying on duality, can be adapted. It also gives a similar new conceptual insight to protomodularity of a regular category having least subobjects, as does Corollary 2.4 to homological categories.

Examples 2.6 (Modular semiexact categories). Another large class of examples of a diagram chasing context is provided by the pairs of the covariant and the contravariant pointed normal subobject functors of modular semiexact categories in the sense of Grandis [10]. These include all abelian categories, as well as the category of graded abelian groups, the categories of projective spaces and projective linear maps over a field, and others - see $[\mathbf{1 1}, \mathbf{1 2}]$. We will now recall a definition for these categories, although we will treat the present topic somewhat briefly since here we do not have any major new remarks to make.

The concept of a semiexact category, due to Grandis, has its origins in $[\mathbf{9}, \mathbf{2 2}]$. Intuitively, it is a category $\mathbb{C}$ equipped with a structure that allows to define kernels and cokernels abstractly, relative to the structure. As shown in [13], such structure can be equivalently given as a full and faithful functor

$$
\mathbb{C}<\mathbb{D},
$$

which has a left adjoint and a right adjoint, with the counit of the first adjunction being a monomorphism admitting pullbacks along arbitrary morphisms, and the unit being an identity, and dually, the unit of the second adjunction being an epimorphism admitting pushouts along arbitrary morphisms, and counit an identity. These pullbacks and pushouts allow to define kernels and cokernels, while the category $\mathbb{D}$ represents the subcategory of null objects of a semiexact category. For instance, when $\mathbb{D}$ above is a singleton category, we recover the context of a pointed category with its usual notions of kernel and cokernel. See the above reference for further details.

The structure of a semiexact category can be also equivalently presented, in some sense, in a more "projective" way, as a functor

$$
\mathbb{B} \longrightarrow \mathbb{C}
$$

having suitable (self-dual) properties — see [20]. In particular, $\mathbb{B}$ can be obtained as the category of "short exact sequences" in $\mathbb{C}$, and then the projection $\mathbb{B} \rightarrow \mathbb{C}$ assigns to the short exact sequence the middle object in the sequence. Equivalently, the functor $\mathbb{B} \rightarrow \mathbb{C}$ is the Grothendieck bifibration of "normal subobjects" in $\mathbb{C}$. It arises via the standard Grothendieck construction from the "transfer functor" in the sense of Grandis, which assigns to each object the lattice of its normal subobjects. By assigning to an object $X$ in $\mathbb{C}$ the pointed set whose elements are objects in the fibre at $X$, with the initial object of the fibre as the base point, we obtain what we call the covariant pointed normal subobject functor of the semiexact category. The contravariant pointed normal subobject functor of the semiexact category is defined dually, by selecting the 
terminal object, instead of the initial object, as the base point. The values of these two functors at morphisms are defined, as expected, via cocartesian and cartesian liftings (in the sense of the theory of Grothendieck fibrations), respectively, i.e., they are given by direct and inverse images of normal subobjects along morphisms. Equivalently, we can get these two functors by composing the "transfer functor" of Grandis $[\mathbf{1 0}, \mathbf{1 2}]$ with suitable forgetful functors.

A semiexact category is modular when the change of base adjunctions between the fibres of the above functor are "modular connections" in the sense of [10] or, equivalently, "exact connections" in the sense of [12]. The property that defines such adjunctions is in fact the same as what in the proof of Theorem 2.2 we referred to as "cartesianness" - in general, as defined in [19], an adjunction is said to be cartesian when the unit of the adjunction is co-cartesian (i.e., the naturality square is a pushout), and the counit of the adjunction is cartesian (i.e., the naturality square is a pullback). A principal example of a modular semiexact category is given by the category of modular lattices with cartesian adjunctions as morphisms.

Similar arguments as those used in the proof of Theorem 2.2 can be used to show that the covariant and the contravariant pointed normal subobject functors of a modular semiexact category give rise to a diagram chasing context (see also Remark 2.8 below). Strictness of this diagram chasing context identifies precisely the Grandis exact categories $[\mathbf{1 0}, \mathbf{1 1}, \mathbf{1 2}]$, which encompass many examples of modular semiexact categories. Most of these examples are actually pointed categories. Note that pointed Grandis exact categories are the same as "quasi exact categories" introduced by Puppe [27], which are also the same as exact categories in the sense of Mitchell [26]. Note also that abelian categories are precisely those pointed Grandis exact categories which have finite products. Examples of (pointed) modular semiexact categories which are not Grandis exact are given by the categories of topological vector spaces over topological fields, as mentioned in [12] (see also Example 2.9 and Remark 2.10 below).

Remark 2.7 (Applications). The present paper gives, in some sense, a simpler way of proving some of the standard diagram lemmas in well-established abstract nonabelian contexts, than what appears in the literature (see $[\mathbf{6}, \mathbf{4}, \mathbf{1 7}, \mathbf{1 8}]$ for proofs of diagram lemmas in sequentiable/homological categories, and see $[\mathbf{1 0}, \mathbf{1 2}]$ for those in modular semiexact categories). In fact, even in particular instances of these contexts, where objects are concrete mathematical structures, such as, say, (non-abelian) groups (which, by the way, form a homological category, but not a modular semiexact category), the approach of the present paper is still in some sense simpler than the more elementary approach based on chasing actual elements of structures, as the latter relies on operating on elements, and does not make use of duality. Unlike the context of a homological category in the sense of $[4]$, the context of a modular semiexact category is self-dual, like that of an abelian category, and the proofs of diagram lemmas in this context, given in $[\mathbf{1 0}, \mathbf{1 2}]$, also rely on duality. The method outlined in this paper still provides in some sense more basic way of proving these diagram lemmas as it does not use explicitly the ordering of subobjects. On the other hand, the advantage of the method used in $[\mathbf{1 0}, \mathbf{1 2}]$ is that the entire diagram chasing argument is reduced to a single chain of equalities of expressions involving meets and joins of subobjects (which, in some sense, play an analogous role as addition and 
subtraction when chasing elements of, say, abelian groups), and so this method is complementary to what the approach of the present paper aims, which is to simplify diagram chasing by avoiding operations.

Remark 2.8 (Generalization). Both the modular semiexact categories, and protomodular categories with least subobjects, are particular instances of a category with a specified "locally bounded biform" in the sense of [20], which is at the same time "cartesian" in the sense of [19]. A cartesian locally bounded biform over a category $\mathbb{C}$ is a faithful Grothendieck bifibration over it, whose fibres are bounded posets, and where the change of base adjunctions between fibres are cartesian in the sense described in Examples 2.6. Any cartesian locally bounded biform over a category $\mathbb{C}$ gives rise to a diagram chasing context by regarding the fibres as pointed sets in two different ways - one with the bottom element being the base point, and the other with the top element being the base point. Diagram chasing contexts obtained in this way include those obtained from protomodular categories with least subobjects and those obtained from modular semiexact categories. We must also remark that from the proof of Theorem 2.2 one can easily extract a similar result on locally bounded biforms, of which Theorem 2.2 would be a corollary; we leave this as an exercise for a reader who is interested in this generalization.

Example 2.9 (Diagram chasing context formed by bounded intervals of a modular lattice). Consider a lattice $L$, and consider the order relation $O$ on $L$, seen as a subset $O \subseteq L \times L$. In fact, $O$ is a sublattice of the cartesian product $L \times L$ of lattices. We can then regard $O$ as a category. Consider the functor $(-)_{*}: O \rightarrow$ Set $_{*}$ which assigns to an object $a=\left(a_{0}, a_{1}\right) \in O$ the pointed set $a_{*}=\left(\left\{x \in L \mid a_{0} \leqslant x \leqslant a_{1}\right\}, a_{0}\right)$, and to a morphism $a \rightarrow b$ the mapping $x \mapsto x \vee b_{0}$ from $a_{*}$ to $b_{*}$. Note that a morphism $a \rightarrow b$ amounts to the inequalities $a_{0} \leqslant b_{0}$ and $a_{1} \leqslant b_{1}$, and so this mapping preserves the base point, $a_{0} \mapsto a_{0} \vee b_{0}=b_{0}$. A dual construction yields a functor $(-)^{*}: O^{\mathrm{op}} \rightarrow \mathbf{S e t}_{*}$ which assigns to an object $a$ the pointed set $\left(\left\{x \in L \mid a_{0} \leqslant x \leqslant a_{1}\right\}, a_{1}\right)$, and to a morphism $a \rightarrow b$ the mapping $x \mapsto x \wedge a_{1}$. We will now show that these two functors constitute a diagram chasing context if and only if the original lattice $L$ is a modular lattice. For a sequence

$$
\left(a_{0}, a_{1}\right) \stackrel{u}{\longrightarrow}\left(b_{0}, b_{1}\right) \stackrel{v}{\longrightarrow}\left(c_{0}, c_{1}\right)
$$

of morphisms in $O$, we have:

$$
\begin{gathered}
\operatorname{Im} u_{*}=\left\{x \vee b_{0} \mid a_{0} \leqslant x \leqslant a_{1}\right\}, \\
\operatorname{Ker} v_{*}=\left\{y \mid b_{0} \leqslant y \leqslant b_{1} \text { and } y \vee c_{0}=c_{0}\right\}=\left\{y \mid b_{0} \leqslant y \leqslant b_{1} \wedge c_{0}\right\}, \\
\operatorname{Im} v^{*}=\left\{z \wedge b_{1} \mid c_{0} \leqslant z \leqslant c_{1}\right\}, \\
\operatorname{Ker} u^{*}=\left\{y \mid b_{0} \leqslant y \leqslant b_{1} \text { and } y \wedge a_{1}=a_{1}\right\}=\left\{b \mid b_{0} \vee a_{1} \leqslant y \leqslant b_{1}\right\} .
\end{gathered}
$$

We then get that exactness of the sequence

$$
a_{*} \stackrel{u_{*}}{\longrightarrow} b_{*} \stackrel{v_{*}}{\longrightarrow} c_{*}
$$

implies $a_{1} \vee b_{0}=b_{1} \wedge c_{0}$, as does exactness of the sequence

$$
c^{*} \stackrel{v^{*}}{\longrightarrow} b^{*} \stackrel{u^{*}}{\longrightarrow} a^{*} .
$$


Under modularity, the equality $a_{1} \vee b_{0}=b_{1} \wedge c_{0}$ would be equivalent to exactness of both sequences. Indeed, this equality readily gives $\operatorname{Im} u_{*} \subseteq \operatorname{Ker} v_{*}$. For the converse inclusion, let $y$ be such that $b_{0} \leqslant y \leqslant b_{1} \wedge c_{0}$. Then $a_{0} \leqslant a_{1} \wedge b_{0} \leqslant a_{1} \wedge y \leqslant a_{1}$. By modularity, $b_{0} \vee\left(a_{1} \wedge y\right)=\left(b_{0} \vee a_{1}\right) \wedge y$. Since $a_{1} \vee b_{0}=b_{1} \wedge c_{0}$, we can conclude $b_{0} \vee$ $\left(a_{1} \wedge y\right)=y$. This shows $\operatorname{Ker} v_{*} \subseteq \operatorname{Im} u_{*}$, as desired. A dual argument will show that modularity implies $\operatorname{Im} v^{*}=\operatorname{Ker} u^{*}$. So, if $L$ is a modular lattice, then (D1) holds. After this, (D2) can be verified straightforwardly. In fact, the equivalence of (a) and (c), and, (d) and (f) follows from the fact that for any morphism $f: a \rightarrow b$, the induced maps $f_{*}$ and $f^{*}$ constitute a Galois connection between two sublattices of $L$,

$$
\left\{x \in L \mid a_{0} \leqslant x \leqslant a_{1}\right\} \rightleftarrows\left\{y \in L \mid b_{0} \leqslant x \leqslant b_{1}\right\},
$$

while the rest of (D2) follows from (D1) and the fact that for any object $a \in O$ we can find a null morphism into $a$ and from $a$ - indeed, for instance the morphisms $\left(a_{0}, a_{0}\right) \rightarrow a$ and $a \rightarrow\left(a_{1}, a_{1}\right)$, respectively (see Remark 1.6). Conversely, to deduce modularity from (D1) and (D2), it suffices to apply (D2) in the special case of a morphism $f:(a \wedge b, a) \rightarrow(b, a \vee b)$ where this time $a$ and $b$ are arbitrary elements of $L$. For this morphism, the sequences

$$
\begin{gathered}
\mathbf{0} \longrightarrow(a \wedge b, a)_{*} \stackrel{f_{*}}{\longrightarrow}(b, a \vee b)_{*}, \\
\mathbf{0} \longrightarrow(b, a \vee b)^{*} \stackrel{f^{*}}{\longrightarrow}(a \wedge b, a)^{*}
\end{gathered}
$$

are exact, and so by (D2), $f_{*}$ must be bijective. Since $f_{*}, f^{*}$ constitute a Galois connection, this means that the maps $f_{*}$ and $f^{*}$ are inverses of each other. So as a consequence of (D2), we can deduce the famous diamond isomorphism theorem in $L$, which is well known to be equivalent to modularity of $L$.

Thus, a modular lattice gives rise to a diagram chasing context. A sequence

$$
a \stackrel{u}{\longrightarrow} b \stackrel{v}{\longrightarrow} a
$$

of morphisms in this diagram chasing context is exact when $a_{1} \vee b_{0}=b_{1} \wedge c_{0}$. A morphism $f: a \rightarrow b$ is a null morphism when $a_{1} \leqslant b_{0}$, and it is an equivalence when $a_{0}=a_{1} \wedge b_{0}$ and $b_{1}=a_{1} \vee b_{0}$. This diagram chasing context is a strict diagram chasing context if and only if the lattice $L$ has at most one element.

Remark 2.10 (Generalization). The above diagram chasing context of a modular lattice is in fact a particular instance of a diagram chasing context arising from a modular semiexact category - in the projective presentation of this modular semiexact category, the "projection" is the composition functor

$$
\mathrm{O} \times{ }_{L} \mathrm{O} \longrightarrow \mathrm{O}
$$

of the modular lattice $L$ seen as a category (the category $O$ then becomes the category of morphisms in $L$ ). This then naturally gives rise to the question of considering any category $\mathbb{C}$ in the place of the lattice $L$ - we leave this for future investigation, only remarking here that the composition functor $\mathbb{C}^{2} \times_{\mathbb{C}} \mathbb{C}^{2} \rightarrow \mathbb{C}^{2}$, where $\mathbb{C}^{2}$ denotes the category of morphisms in $\mathbb{C}$, is a Grothendieck bifibration when $\mathbb{C}$ has pullbacks and pushouts, and requiring the change of base adjunctions of this bifibration to be cartesian brings us close to the notion of a modular category due to Carboni [7]. 
Example 2.11 (Diagram chasing context formed by elements of a Boolean algebra). In all examples of a diagram chasing context we encountered so far, for any morphism $f$, the maps $f_{*}$ and $f^{*}$ underly an adjunction between posets. This need not be always the case, as we will now show, albeit with a somewhat artificial example. In it, the pair of functors for the diagram chasing context are in fact identical to each other.

Consider a Heyting algebra $\mathcal{H}$, regarded as a single object category whose morphisms are elements of $\mathcal{H}$, and composition is given by the meet operation of the Heyting algebra. For the unique object $X$ in $\mathbb{C}$, define $X_{*}=X^{*}$ to be the underlying set of the given Heyting algebra, with the bottom element 0 as the base point. Now for two elements $a, b \in \mathcal{H}$, define

$$
a_{*}(b)=a^{*}(b)=a \wedge b .
$$

Then for a sequence

$$
X \stackrel{u}{\longrightarrow} X \stackrel{v}{\longrightarrow} X
$$

we have

$$
\begin{gathered}
\operatorname{Im} u_{*}=\{b \mid b \leqslant u\}, \\
\operatorname{Ker} v_{*}=\{b \mid v \wedge b=0\}=\{b \mid b \leqslant v \rightarrow 0\}, \\
\operatorname{Im} v^{*}=\{b \mid b \leqslant v\}, \\
\operatorname{Ker} u^{*}=\{b \mid u \wedge b=0\}=\{b \mid b \leqslant u \rightarrow 0\}
\end{gathered}
$$

and so exactness of the sequence

$$
X_{*} \stackrel{u_{*}}{\longrightarrow} X_{*} \stackrel{v_{*}}{\longrightarrow} X_{*}
$$

is equivalence to $u=v \rightarrow 0$, while exactness of the sequence

$$
X^{*} \longleftarrow u^{*} X^{*} \longleftarrow v^{*} X^{*}
$$

is equivalent to $v=u \rightarrow 0$. This means that (D1) is equivalent to the Heyting algebra being a Boolean algebra. It is easy to see that conditions (a), (c), (d) and (f) in (D2) are each equivalent to $f$ being the top element of the Heyting algebra. So, by Remark 1.6, we get that once (D1) holds also (D2) holds (the bottom element 0 of the Boolean algebra is the unique null morphism). To verify this directly, note that the conditions (b) and (e) in (D2) each state that

$$
f \wedge a=0 \quad \Leftrightarrow \quad a=0
$$

holds for any $a \in \mathcal{H}$. In particular, if we take $a=f \rightarrow 0$, we see that $f \rightarrow 0=0$. This is in fact not only necessary but also sufficient to have the above equivalence for every $a \in \mathcal{H}$, since $f \wedge a=0$ is equivalent to $a \leqslant f \rightarrow 0$. In a Boolean algebra, $f \rightarrow 0=0$ is equivalent to $f$ being the top element, which completes what we wanted to show. Finally, note that (D3) always holds, since the map $f \wedge(-)$ is a bijection if and only if $f$ is the top element of the Heyting algebra. Thus, we get a diagram chasing context if and only if we get a strict one, and if and only if the Heyting algebra is a Boolean 
algebra. When this is the case, an exact sequence is a sequence of the form

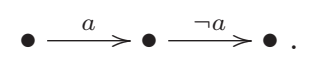

Since, as remarked above, the bottom element 0 of the Boolean algebra is the unique null morphism, the unique object is a null object if and only if the Boolean algebra is trivial, i.e., $1=0$.

\section{References}

[1] M. Barr, P.A. Grillet and D.H. van Osdol, Exact Categories and Categories of Sheaves, Lecture Notes in Math. 236, Springer-Verlag, 1971.

[2] S. Mac Lane and G. Birkhoff, Algebra (third edition), Chelsea Publishing Co., New York, 1988.

[3] F. Borceux, An Axiomatic Approach to Geometry (Geometric Trilogy I), Springer, 2014.

[4] F. Borceux and D. Bourn, Mal'cev, Protomodular, Homological and SemiAbelian Categories, Math. Appl. 566, Kluwer, 2004.

[5] D. Bourn, Normalization equivalence, kernel equivalence and affine categories, Lecture Notes in Math. 1488 (1991), 43-62.

[6] D. Bourn, 3×3 lemma and protomodularity, J. Algebra 236 (2001), 778-795.

[7] A. Carboni, Categories of affine spaces, J. Pure Appl. Algebra 61 (1989), 243250 .

[8] S. Eilenberg and S. Mac Lane, General theory of natural equivalences, Trans. Amer. Math. Soc. 58 (1945), 231-295.

[9] C. Ehresmann, Sur une notion générale de cohomologie, C. R. Acad. Sci. Paris 259 (1964), 2050-2053.

[10] M. Grandis, On the categorical foundations of homological and homotopical algebra, Cah. Topol. Géom. Différ. Catég. 33 (1992), 135-175.

[11] M. Grandis, Homological Algebra, the Interplay of Homology with Distributive Lattices and Orthodox Semigroups, World Scientific Publishing Co., Singapore, 2012 .

[12] M. Grandis, Homological Algebra in Strongly Non-Abelian Settings, World Scientific Publishing Co., Singapore, 2013.

[13] M. Grandis, G. Janelidze and L. Márki, Non-pointed exactness, radicals, closure operators, J. Aust. Math. Soc. 94 (2013), 348-361.

[14] A. Grothendieck, Technique de descente et théorèmes d'existence en géométrie algébrique. I. Généralités. Descente par morphismes fidèlement plats, Séminaire Bourbaki Exp. No. 190 (1959), 299-327.

[15] A. Grothendieck, Catégories fibrées et descente, Exposé VI, in: Revêtements Etales et Groupe Fondamental (SGA1), Lecture Notes in Math. 224 (1971), 145-194.

[16] G. Janelidze, L. Márki and W. Tholen, Semi-abelian categories, J. Pure Appl. Algebra 168 (2002), 367-386. 
[17] Z. Janelidze, The pointed subobject functor, $3 \times 3$ lemmas, and subtractivity of spans, Theory Appl. Categ. 23 (2010), 221-242.

[18] Z. Janelidze, An axiomatic survey of diagram lemmas for non-abelian grouplike structures, J. Algebra 370 (2012), 387-401.

[19] Z. Janelidze, On the form of subobjects in semi-abelian and regular protomodular categories, Appl. Categ. Structures 22 (2014), 755-766.

[20] Z. Janelidze and T. Weighill, Duality in non-abelian algebra I. From cover relations to Grandis ex2-categories, Theory Appl. Categ. 29 (2014), 315-331.

[21] Z. Janelidze and T. Weighill, Duality in non-abelian algebra II. From Isbell bicategories to Grandis exact categories, to appear in J. Homotopy Relat. Struct. DOI 10.1007/s40062-015-0116-9

[22] R. Lavendhomme, La notion d'idéal dans la théorie des catégories, Ann. Soc. Sci. Bruxelles Sér. 179 (1965), 5-25.

[23] S. Mac Lane, Duality for groups, Bull. Amer. Math. Soc. 56 (1950), 485-516.

[24] S. Mac Lane, Homology, Springer-Verlag, 1963.

[25] S. Mac Lane, Categories for the Working Mathematician (second edition), Grad. Texts in Math. 5, Springer-Verlag, 1998.

[26] B. Mitchell, Theory of Categories, Academic Press, 1965.

[27] D. Puppe, Korrespondenzen in abelschen Kategorien, Math. Ann. 148 (1962), $1-30$.

[28] O. Veblen and J. W. Young, Projective Geometry (Volume 1), Ginn and Company, 1910.

[29] O. Wyler, Weakly exact categories, Arch. Math. (Basel) 17 (1966), 9-19.

Zurab Janelidze zurab@sun.ac.za

Mathematics Division, Department of Mathematical Sciences, Stellenbosch University, Private Bag X1 Matieland 7602, South Africa 